\title{
The interferon-inducible antiviral protein Daxx is not essential for interferon-mediated protection against avian sarcoma virus
}

\author{
Kelsey A Haugh, Natalia Shalginskikh, Shoko Nogusa, Anna Marie Skalka, Richard A Katz \\ and Siddharth Balachandran*
}

\begin{abstract}
Background: The antiviral protein Daxx acts as a restriction factor of avian sarcoma virus (ASV; Retroviridae) in mammalian cells by promoting epigenetic silencing of integrated proviral DNA. Although Daxx is encoded by a type I ( $(/ / \beta)$ interferon-stimulated gene, the requirement for Daxx in the interferon anti-retroviral response has not been elucidated. In this report, we describe the results of experiments designed to investigate the role of Daxx in the type I interferon-induced anti-ASV response.
\end{abstract}

Findings: Using an ASV reporter system, we show that type I interferons are potent inhibitors of ASV replication. We demonstrate that, while Daxx is necessary to silence ASV gene expression in the absence of interferons, type I interferons are fully-capable of inducing an antiviral state in the absence of Daxx.

Conclusions: These results provide evidence that Daxx is not essential for the anti-ASV interferon response in mammalian cells, and that interferons deploy multiple, redundant antiviral mechanisms to protect cells from ASV.

Keywords: Daxx, Interferon, Avian sarcoma virus, Innate immunity

\section{Findings}

Introduction

Avian Sarcoma Virus (ASV) is a prototypic alpharetrovirus (family Retroviridae) that can be pseudotyped to transduce mammalian cells and study host antiviral responses. We have previously shown that the cellular scaffolding protein Daxx, originally identified a mediator of death-receptortriggered apoptosis [1], is also a potent anti-ASV restriction factor [2]. Daxx is recruited to viral DNA by ASV integrase, where it promotes the rapid epigenetic repression of integrated viral DNA via recruitment of generepressive histone deacetylases (HDACs) and DNA methyl transferases [2,3]. We identified an essential role for Daxx in controlling ASV replication by demonstrating that genetic ablation or RNA interference-mediated knockdown of Daxx expression resulted in significantly-increased expression of an ASV-encoded reporter gene [2,3].

\footnotetext{
*Correspondence: richard.katz@fccc.edu; siddharth.balachandran@fccc.edu Immune Cell Development and Host Defense Program, Fox Chase Cancer Center, Room 422 Reimann Building, 333 Cottman Ave., 19111 Philadelphia, PA, USA
}

\section{() BioMed Central}

Type I (predominantly $\alpha / \beta$ ) interferons (IFNs), are a family of cytokines with powerful antiviral and immunemodulatory effects, and are rapidly induced in most cells upon virus infection. Once produced, IFNs activate an antiviral state in the infected cell, as well as in surrounding cells, by Jak/STAT-regulated induction of $>1000$ IFNstimulated genes (ISGs) [4,5]. Daxx mRNA and protein expression are induced following exposure to type I IFNs, indicating that Daxx is an ISG $[2,3]$.

Here, we demonstrate that type I IFNs are powerful inhibitors of ASV replication in human and avian cells. We show that, although Daxx is upregulated by type I IFNs and essential on its own for silencing ASV gene expression in human cells, it is largely dispensable for establishment of the type I IFN-induced anti-retroviral state. Our results suggest that IFNs are capable of effectively inhibiting ASV even in the absence of Daxx, providing evidence that epigenetic silencing by Daxx is a redundant mechanism of the IFN anti-ASV response in mammalian cells. 
Type I IFNs inhibit ASV replication in mammalian cells

To investigate whether type I IFNs can block the early steps in ASV replication, we treated HeLa cells with either human IFN- $\alpha$ or IFN- $\beta$ prior to infection with an ASV-GFP reporter virus. This reporter virus is pseudotyped to express the murine leukemia virus (MuLV) amphotropic envelope protein, and is therefore capable of entry into mammalian cells. ASV-GFP contains an intact complement of replicative genes, and is fully-capable of productive infection in its natural avian host cells, but several post-transcriptional blocks in mammalian cells inhibit late events in the virus life-cycle, limiting infection to a single round in these cells [2,3]. ASV-GFP infection of mammalian cells, however, recapitulates key early events of the retroviral life-cycle, including entry, uncoating, reverse-transcription and integration. As diminished GFP expression is a faithful readout of Daxx-dependent silencing, we have previously employed ASV-GFP to identify post-integration silencing of retroviral gene-expression as a Daxx-sensitive step [2,3].

After treating HeLa cells with either IFN- $\alpha$ or IFN- $\beta$ for $18 \mathrm{~h}$, we infected these cells with ASV-GFP in the presence of DEAE-Dextran $(20 \mu \mathrm{g} / \mathrm{mL})$, as described previously [6], and quantified viral gene expression by measuring GFP fluorescence $48 \mathrm{~h}$ post-infection. As the IFN-induced antiviral state is rarely maintained for more than $30 \mathrm{~h}$ post-treatment [7], cells were supplemented with IFN $6 \mathrm{~h}$ and $24 \mathrm{~h}$ post infection. Vesicular stomatitis virus encoding GFP (VSV-GFP) [8] was used as a positive control for IFN activity, as VSV is a well-established IFNsensitive virus $[9,10]$. We found that treatment of HeLa cells with either IFN- $\alpha$ or IFN- $\beta$ efficiently diminished GFP positivity (by $\sim 70 \%$ and $\sim 85 \%$, respectively) following ASV infection, demonstrating that type I IFNs are capable of blocking ASV gene expression (Figure 1A,B). As expected, IFN- $\alpha$ and IFN- $\beta$ inhibited VSV-GFP replication almost completely (from $>75 \%$ GFP-positive cells in untreated controls to $<1 \%$ GFP-positive cells after IFN- $\alpha / \beta$ treatment; Figure 1C,D).

\section{Type I IFNs Inhibit ASV replication in avian cells}

To extend this investigation to cells of natural ASV hosts, we performed similar experiments in DF-1 chicken cells. We limited ASV replication to a single round in these cells by using a self-inactivating ASV-based alpharetroviral GFP-transducing vector with diminished LTR transcriptional activity [11]. After treating DF-1 cells with chicken IFN- $\alpha$ for $18 \mathrm{~h}$, we infected these with $5 \mu \mathrm{L}$ of self-inactivating ASV-GFP in the presence of Polybrene $(10 \mu \mathrm{g} / \mathrm{mL})$ at $37^{\circ} \mathrm{C}$ for $1 \mathrm{~h}$. To ensure continued maintenance of the antiviral state, we supplemented cells with IFN- $\alpha 6 \mathrm{~h}$ and $24 \mathrm{~h}$ p.i. When we examined these cells by GFP-based flow cytometry $48 \mathrm{~h}$ p.i., we observed that treatment with chicken type I IFN diminished proviral

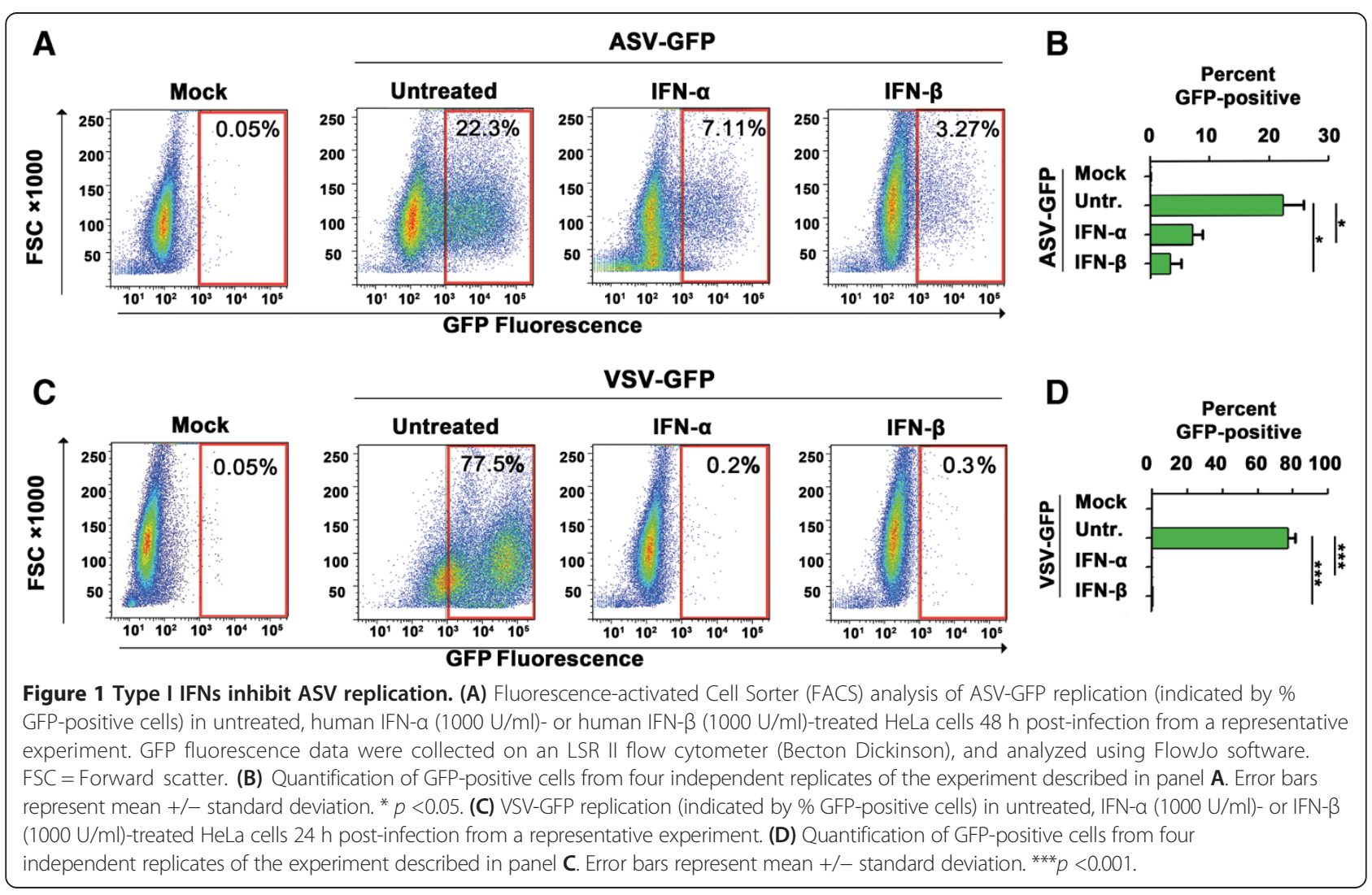


reporter gene expression by a significant amount (by $~ 70 \%$, Figure 2), as observed in mammalian cells (Figure 1A-D). Collectively, these results demonstrate that type I IFNs exert antiviral activity against ASV, and set the stage for experiments designed to determine if Daxx is an essential component of the IFN anti-ASV program.

\section{Daxx is induced by type I IFNs in mammalian and avian cells}

We previously demonstrated that treatment with IFN- $\alpha$ results in induction of Daxx mRNA in HeLa cells [3]. To evaluate Daxx protein levels following IFN treatment, we treated HeLa or DF-1 cells with either human or chicken IFN- $\alpha$, respectively, and examined whole-cell lysates prepared from these cells at various times posttreatment by immunoblotting. As shown in Figure 3A, IFN treatment increased Daxx protein levels $\sim 3$-fold by $24 \mathrm{~h}$ in HeLa cells. In DF-1 cells, IFN- $\alpha$ induction of Daxx was confirmed to occur at the mRNA level $(\sim 2.5-$ fold, Figure 3B). A protein band of the approximate size of the putative avian Daxx ortholog was similarly induced by chicken IFN- $\alpha$ (Figure $3 C$ ). Thus, Daxx is an IFNinducible protein in both mammalian and avian cells.

\section{Daxx is not essential for type I IFN-mediated inhibition of ASV replication in mammalian cells}

To directly determine if Daxx contributed non-redundantly to the IFN-induced antiviral state in mammalian cells, we knocked-down Daxx expression by RNAi. Individual or pooled transfection of HeLa cells with four distinct siRNAs that target Daxx mRNA reduced Daxx protein levels in HeLa cells to nearly-undetectable levels within $72 \mathrm{~h}$ of treatment (Figure 4A). By contrast, Daxx levels in cells transfected with control non-silencing siRNAs were comparable to those seen in untreated HeLa cells (Figure 4A). To determine whether Daxx knockdown resulted in an altered ISG induction profile following IFN treatment, we transfected HeLa cells with control or pooled Daxx
siRNAs, treated these cells with IFN- $\alpha$, and analyzed lysates from these cells by immunoblotting. Knockdown of Daxx did not significantly alter the kinetics or magnitude of STAT1 phosphorylation by IFN- $\alpha$ (Figure 4B). Over a longer time course of $24 \mathrm{~h}$, Daxx knockdown did not affect the kinetics of induction of prototypic ISGencoded proteins STAT1 and MX1, and only modestly affected the magnitude of their induction (Figure 4C). Together, these results indicate that loss of Daxx does not significantly alter IFN- $\alpha$-induced transcription.

To test if Daxx was required for IFN-mediated antiviral activity against ASV, we first transfected HeLa cells with either non-silencing siRNAs, or with a pool of the four siRNAs that target Daxx, and exposed these cells to IFN- $\alpha$ or IFN- $\beta$ 54 h post-transfection. At $72 \mathrm{~h}$ postsiRNA transfection (and $18 \mathrm{~h}$ post IFN treatment) when Daxx knockdown was maximal (Figure 4A), cells were infected with ASV-GFP and supplemented with IFN $6 \mathrm{~h}$ and $24 \mathrm{~h}$ p.i., before analysis by flow cytometry at $48 \mathrm{~h}$ p.i. Consistent with previous findings, ASV reporter gene expression was higher in Daxx-knockdown cells compared to those treated with nonspecific siRNA (by about twofold, Figure 4D,E). However, both IFN- $\alpha$ and IFN- $\beta$ reduced ASV-GFP expression to approximately the same levels ( $\sim 5 \%$ by IFN- $\alpha$ and $\sim 3 \%$ by IFN- $\beta$ ) in cells transfected with siRNA to Daxx as they did in cells carrying non-silencing siRNAs (Figure 4D,E). These results provide evidence that Daxx is not essential for IFN-mediated protection against ASV in mammalian cells.

\section{Conclusions}

The two salient findings of this study are that (1) type I IFNs can potently inhibit ASV replication in mammalian and avian cells, and (2) that although Daxx is IFNinducible, IFN-mediated anti-ASV activity in mammalian cells does not require Daxx. Together with our previous demonstration that Daxx is essential for anti-retroviral host defense in the absence of IFNs [2,3], our current
A

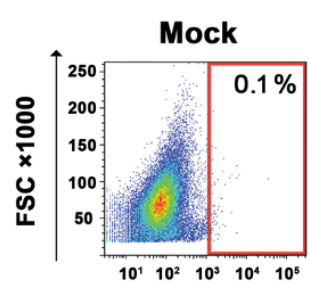

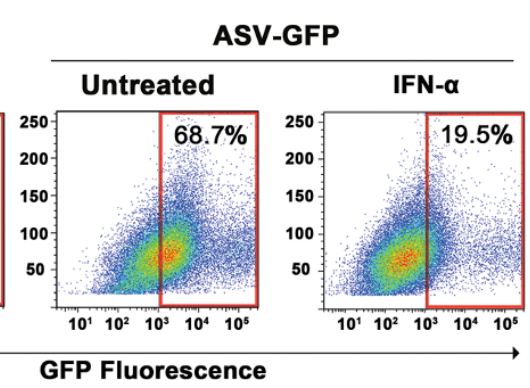

GFP Fluorescence
B

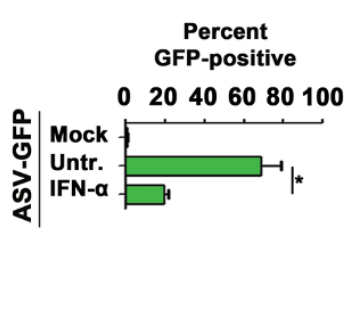

Figure 2 Chicken IFN-a inhibits ASV replication in DF-1 cells. (A) FACS analysis of ASV-GFP replication (indicated by \% GFP-positive cells) in untreated or chicken IFN-a (1000 U/ml)-treated DF-1 cells $48 \mathrm{~h}$ post-infection from a representative experiment. FSC = Forward scatter. (B) Quantification of GFP-positive cells from three independent replicates of the experiment described in panel $\mathbf{A}$. Error bars represent mean $+/-$ standard deviation. ${ }^{*} p<0.05$. 


Figure 3 Daxx is induced by type I IFNs. (A) Immunoblot analysis of Daxx protein levels (antibody from Sigma) after human (h) IFN-a (1000 U/ml)
treatment of HeLa cells for the indicated time points. (B) Real-time PCR showing Daxx mRNA levels in DF-1 cells following treatment with chicken (ch)
IFN-a for the indicated time points. Values were normalized to GAPDH. (C) Immunoblot analysis of Daxx protein levels following chicken (ch) IFN-a
treatment of DF-1 cells for the indicated times. $\beta$-actin (antibody from Sigma) is shown as a loading control. Molecular-weight markers, in kilodaltons,
are shown to the left of each blot.

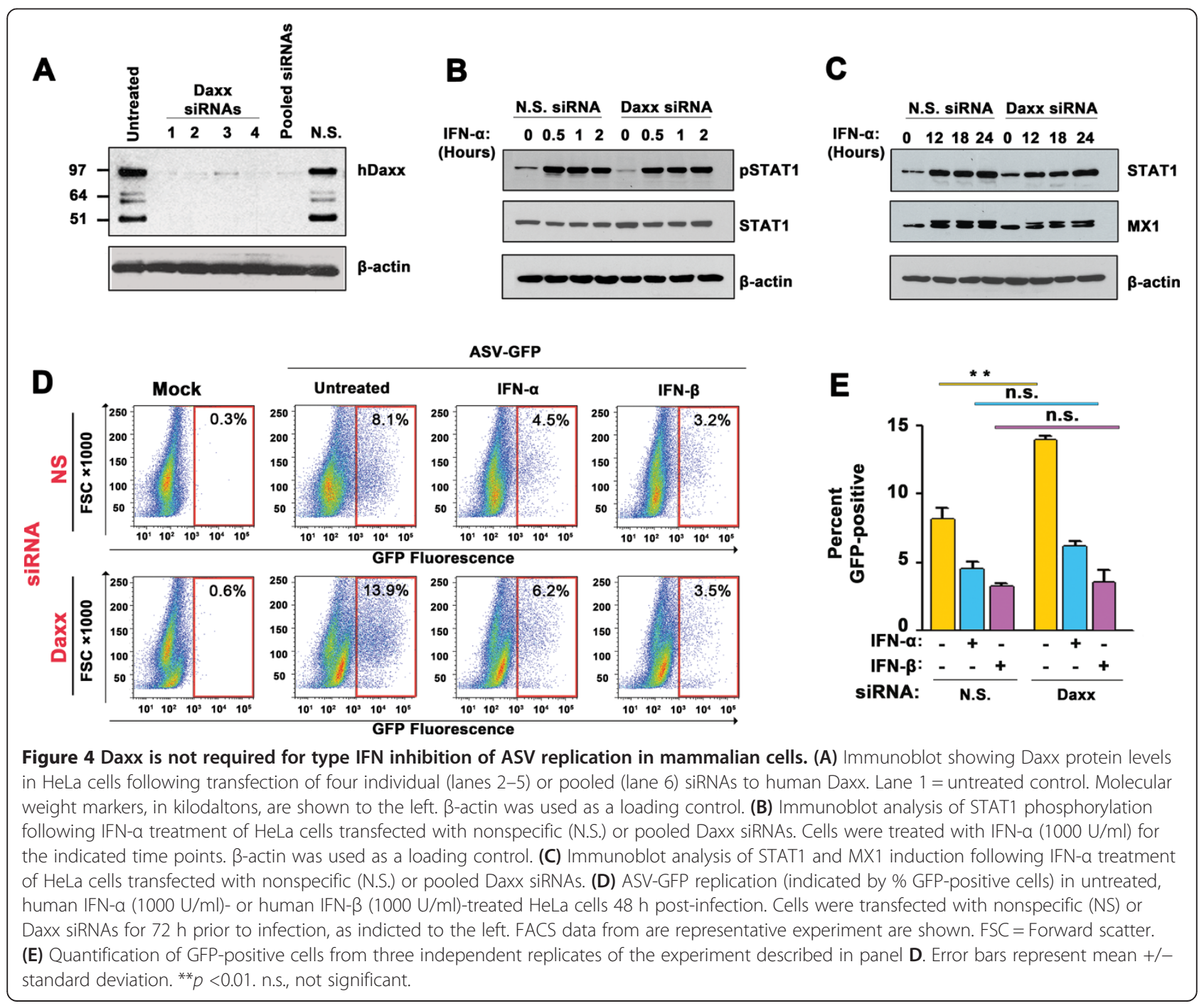


observations support a model for Daxx function in which Daxx protects against ASV (by epigenetic repression of ASV proviral gene expression) prior to induction of IFNs. Once induced, type I IFNs can establish an anti-retroviral state in which Daxx is not essential.

A Daxx ortholog has been identified in Drosophila and other insects [12], predating by $\sim 150$ million years the emergence of IFN- $\alpha / \beta$ genes, which can be traced back $\sim 250$ million years to the time when reptiles and birds diverged from each other [13,14]. It is thus possible that Daxx represents an ancient, metazoan anti-retroviral protein the function of which remains essential in the absence of IFNs, but which has since been rendered redundant by the relatively-recent emergence of the type I IFN system in higher vertebrates.

Alternatively, our finding that IFN-mediated protection against ASV is Daxx-independent may be explained simply by activation of mechanistically distinct, but functionally redundant ISGs. Indeed, dependence on a single ISG may be detrimental to the host in the face of a virus infection, as viruses are capable of rapid evolution and consequent subversion of antiviral host proteins. Several viruses are known to target Daxx. For example, the human cytomegalovirus (HCMV) virion tegument protein pp71, as well as the adenovirus E1B- $55 \mathrm{~K}$ protein have been shown to induce degradation of Daxx via the proteasome [15]. Redundant antiviral mechanisms ensure that multiple host defense strategies are in place, should any one, e.g. Daxx, be compromised by virus infection. We speculate that in mammalian cells, IFNs target multiple early steps in the ASV life cycle upstream of where Daxx is proposed to act, including entry, capsid disassembly, uncoating, nuclear entry/reverse transcription, and integration. As IFNs are capable of anti-ASV activity even in the absence of Daxx, epigenetic repression of proviral DNA by Daxx likely represents only one of the many diverse pathways, including those activated by APOBEC3G, TRIM5, TRIM22, and MXB, which are deployed by IFNs to restrict early steps of retroviral replication [16-18]. For example, APOBEC3G triggers damaging hypermutation of retroviral cDNA following reverse transcription, TRIM5 blocks HIV-1 by inhibiting viral cDNA synthesis, and MXB has been reported to inhibit HIV-1 DNA integration [16-18]. Induction of these or similar restriction factors may account for IFN-mediated protection against ASV in the absence of Daxx.

\section{Competing interests}

The authors declare that they have no competing interest.

\section{Authors' contributions}

$\mathrm{KAH}$ performed most of the experiments and wrote the manuscript. NS and SN generated virus stocks and assisted with experiments. RK, AMS, and SB conceived this study and edited the manuscript. All authors read and approved the final manuscript.

\section{Acknowledgements}

This work was supported by an ACS Research Scholar Grant (RSG-09-195-01 $M P C$ ), and National Institutes of Health grant R21Al104212 (SB), and by a National Institutes of Health grant RO1CA71515 (RK and AMS). Additional support was provided by the W.W. Smith Charitable Trust (SB and AMS), and by the F.M. Kirby Foundation (SB). The funding bodies had no role in the design, collection, analysis, and interpretation of data; in the writing of the manuscript; and in the decision to submit the manuscript for publication.

Received: 3 February 2014 Accepted: 23 May 2014

Published: 28 May 2014

\section{References}

1. Yang X, Khosravi-Far R, Chang HY, Baltimore D: Daxx, a novel Fas-binding protein that activates JNK and apoptosis. Cell 1997, 89:1067-1076.

2. Greger JG, Katz RA, Ishov AM, Maul GG, Skalka AM: The cellular protein daxx interacts with avian sarcoma virus integrase and viral DNA to repress viral transcription. J Virol 2005, 79:4610-4618.

3. Shalginskikh N, Poleshko A, Skalka AM, Katz RA: Retroviral DNA methylation and epigenetic repression are mediated by the antiviral host protein Daxx. J Virol 2013, 87:2137-2150.

4. Platanias LC: Mechanisms of type-I- and type-II-interferon-mediated signalling. Nat Rev Immunol 2005, 5:375-386.

5. Stark GR, Kerr IM, Williams BR, Silverman RH, Schreiber RD: How cells respond to interferons. Annu Rev Biochem 1998, 67:227-264.

6. Schaefer-Klein J, Givol I, Barsov EV, Whitcomb JM, VanBrocklin M, Foster DN, Federspiel MJ, Hughes SH: The EV-O-derived cell line DF-1 supports the efficient replication of avian leukosis-sarcoma viruses and vectors. Virology 1998, 248:305-311.

7. Balachandran S, Thomas E, Barber GN: A FADD-dependent innate immune mechanism in mammalian cells. Nature 2004, 432:401-405.

8. Fernandez M, Porosnicu M, Markovic D, Barber GN: Genetically engineered vesicular stomatitis virus in gene therapy: application for treatment of malignant disease. J Virol 2002, 76:895-904.

9. Balachandran S, Barber GN: Defective translational control facilitates vesicular stomatitis virus oncolysis. Cancer Cell 2004, 5:51-65.

10. Stojdl DF, Lichty BD, enOever BR, Paterson JM, Power AT, Knowles S, Marius R, Reynard J, Poliquin L, Atkins H, Brown EG, Durbin RK, Durbin JE, Hiscott J, Bell JC: VSV strains with defects in their ability to shutdown innate immunity are potent systemic anti-cancer agents. Cancer Cell 2003, 4:263-275.

11. Suerth JD, Maetzig T, Galla M, Baum C, Schambach A: Self-inactivating alpharetroviral vectors with a split-packaging design. J Virol 2010, 84:6626-6635.

12. Santiago A, Godsey AC, Hossain J, Zhao LY, Liao D: Identification of two independent SUMO-interacting motifs in Daxx: evolutionary conservation from Drosophila to humans and their biochemical functions. Cell Cycle 2009, 8:76-87.

13. Roberts RM, Liu L, Guo Q, Leaman D, Bixby J: The evolution of the type I interferons. J Interferon Cytokine Res 1998, 18:805-816.

14. Crawford NG, Faircloth BC, McCormack JE, Brumfield RT, Winker K, Glenn TC: More than 1000 ultraconserved elements provide evidence that turtles are the sister group of archosaurs. Biol Lett 2012, 8:783-786.

15. Schreiner $\mathrm{S}$, Wodrich $\mathrm{H}$ : Virion factors that target Daxx to overcome intrinsic immunity. J Virol 2013, 87:10412-10422.

16. Malim MH, Bieniasz PD: HIV restriction factors and mechanisms of evasion. Cold Spring Harb Perspect Med 2012, 2:a006940.

17. Hattlmann CJ, Kelly JN, Barr SD: TRIM22: a diverse and dynamic antiviral protein. Mol Biol Int 2012, 2012:153415.

18. Liu Z, Pan Q, Ding S, Qian J, Xu F, Zhou J, Cen S, Guo F, Liang C: The interferon-inducible MxB protein inhibits HIV-1 infection. Cell Host Microbe 2013, 14:398-410.

doi:10.1186/1743-422X-11-100

Cite this article as: Haugh et al:: The interferon-inducible antiviral protein Daxx is not essential for interferon-mediated protection against avian sarcoma virus. Virology Journal 2014 11:100. 